PAPER

\section{Gyrokinetic study of turbulence suppression in a JET-ILW power scan}

To cite this article: H Doerk et al 2016 Plasma Phys. Control. Fusion 58115005

View the article online for updates and enhancements.

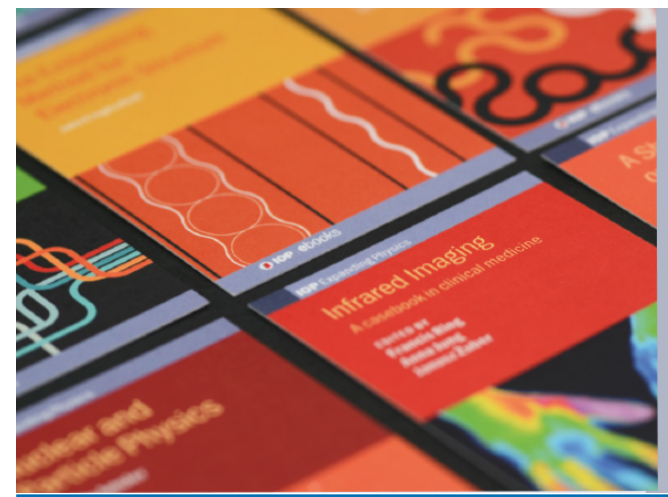

\section{IOP ebooks}

Bringing together innovative digital publishing with leading authors from the global scientific community. Start exploring the collection-download the first chapter of every title for free. 


\title{
Gyrokinetic study of turbulence suppression in a JET-ILW power scan
}

\author{
H Doerk ${ }^{1}$, C Challis ${ }^{2}$, J Citrin ${ }^{3,4}$, J Garcia $^{4}$, T Görler $^{1}$, F Jenko ${ }^{5}$ \\ and JET Contributors ${ }^{6}$ \\ EUROfusion Consortium, JET, Culham Science Centre, Abingdon, OX14 3DB, UK \\ ${ }^{1}$ Max-Planck-Institut für Plasmaphysik, Boltzmannstraße 2, D-85748 Garching, Germany \\ 2 CCFE, Culham Science Centre, Abingdon, OX14 3DB, UK \\ ${ }^{3}$ FOM Institute DIFFER, PO Box 1207 NL-3430 BE Nieuwegein, The Netherlands \\ ${ }^{4}$ CEA, IRFM, F-13108 Saint-Paul-lez-Durance, France \\ ${ }^{5}$ University of California, Los Angeles, CA 90095, USA \\ E-mail: hauke.doerk@ipp.mpg.de
}

Received 21 April 2016, revised 29 July 2016

Accepted for publication 18 August 2016

Published 15 September 2016

\begin{abstract}
For exploring tokamak operation regimes that deliver both high $\beta$ and good energy confinement, power scans at JET with ITER-like wall have been performed. Relatively weak degradation of the confinement time coincides with increased core temperature of the ions at high power. The changes in core turbulence characteristics during a power scan with an optimized (broad) $q$ profile are analyzed by means of nonlinear gyrokinetic simulations. The increase in $\beta$ is crucial for stabilizing ion temperature gradient driven turbulence, accompanied by increased ion to electron temperature ratio, the presence of a dynamic fast ion species, as well as the geometric stabilization by increased thermal and suprathermal pressure. A sensitivity study with respect to the $q$ profile reveals that electromagnetic effects are more pronounced at larger values of $q$. Further, it is confirmed that turbulence suppression due to rotation becomes less effective in such strongly electromagnetic systems. Electrostatic simplified models may thus perform well in present-day devices, in which high $\beta$ is often correlated with high rotation, but provide poor extrapolation towards low rotation devices. Implications for ITER and reactor plasmas are discussed.
\end{abstract}

Keywords: tokamak, gyrokinetic simulation, thermal transport, electromagnetic turbulence

(Some figures may appear in colour only in the online journal)

\section{Introduction}

For realizing adequate fusion power in magnetically confined plasmas, a sufficiently large energy confinement time $\tau_{E}$ is mandatory. At the same time, a high (thermal) normalized plasma pressure $\beta_{\text {th }}=2 \mu_{0} p_{\text {th }} / B^{2}$ must be reached. Experimental realization of both requirements is often accompanied by an optimized (broad) shape of the $q$ profile and low collisionality, in plasma scenarios variously described as 'improved $\mathrm{H}$-mode, 'advanced inductive' or 'hybrid' (e.g. [1-3]). With the aim of understanding the confinement scaling in preparation for

${ }^{6}$ See the appendix of [40].
ITER, a series of four power scans are performed at JET [4], covering low and high triangularity $\delta$, as well as Carbon (C) and ITER-like metal wall (ILW) materials. Applying a 'current overshoot' [3], the 'hybrid' regime is accessed at high power. In the high- $\delta$ ILW case, $\tau_{E}$ remains below the value obtained with $\mathrm{C}$-wall at low heating power. However, the plasma stored energy increases more rapidly with power, so that suitable confinement regimes can be reached also with ILW. In low- $\delta$ shaping, the increase of stored energy with absorbed power is similarly strong for both wall materials. Power degradation of the order of $\tau_{E} \sim P^{-0.3}$ is much weaker than the ITER physics base result $\tau_{I P B 98 y 2} \sim P^{-0.7}$ [5]. Detailed studies exist for high-power C-wall 'hybrid' plasmas at JET, where high 
Table 1. Nominal local parameters at $\rho_{\text {tor }}=0.33$ from CRONOS.

\begin{tabular}{llll}
\hline JET & 84798 & 84792 & 84792 alt. \\
\hline$\nu_{\mathrm{i}}^{\star}$ & 0.0645 & 0.0176 & 0.0106 \\
$1 / \rho^{\star}$ & 305.2 & 245.1 & 242.4 \\
$\beta_{\mathrm{e}}(\%)$ & 1.236 & 2.09 & 2.00 \\
$q$ & 1.1814 & 1.2380 & 0.9152 \\
$\hat{s}$ & 0.1445 & 0.1447 & 0.1762 \\
$R / \mathrm{m}$ & 2.9977 & 3.0578 & 2.9855 \\
$\alpha$ & 0.1753 & 0.4748 & 0.2545 \\
$\alpha_{\mathrm{th}}$ & 0.1539 & 0.3943 & 0.2105 \\
$\gamma_{E \times B} /\left(c_{s} / R\right)$ & 0.1320 & 0.1522 & 0.2311 \\
$Z_{\mathrm{eff}}$ & 1.1590 & 1.2600 & 1.2600 \\
$T_{0 e} / \mathrm{keV}$ & 2.0846 & 3.1663 & 3.1663 \\
$\left\langle Q_{\mathrm{e}} V^{\prime}\right\rangle / \mathrm{MW}$ & 0.408 & 1.4 & 1.4 \\
$\left\langle Q_{\mathrm{i}} V^{\prime}\right\rangle / \mathrm{MW}$ & 1.40 & 2.8 & 2.8 \\
\hline
\end{tabular}

Note: For discharge 84792, mapping to the equilibrium files with $q=1.24$ and $q=0.915$ (alt.) are shown. Whereby the parameters $\gamma_{E}, \alpha$ and $\nu_{\mathrm{i}}^{\star}$ are directly affected by a change in $q$.

plasma $\beta$ plays a crucial role for explaining the measured beneficial power scaling $[6,7]$. Electromagnetic (EM) effectsassociated to finite $\beta$-suppress turbulent heat transport in the plasma core and thus allow steeper temperature profiles. In addition, enhanced pressure increases pedestal MHD stability by the geometric effect of flux compression. These two $\beta$ effects on core and pedestal can reinforce themselves [4, 7], and since both profit from an increased contribution of fast particles $\left(\beta_{\text {fast }}\right)$ at higher power, a positive feedback loop may be initiated.

Regarding core turbulent transport, the effectiveness of EM stabilization is found to be indicated by the ratio $\beta / \beta_{\text {crit }}$, where $\beta$ includes thermal and suprathermal pressure, $[6,8$, 9]. This $\beta$ stabilization is more pronounced in nonlinear than in linear simulations [10] as thoroughly confirmed by benchmarks between several gyrokinetic codes, [11]. The threshold for Alfvénic EM instabilities, $\beta_{\text {crit }}$, generally decreases at low magnetic shear, so that this effect can be favoured by the flat $q$ profiles in the inner half-radius of 'hybrid' discharges. Low power degradation has been found also in baseline power scans [12], though, which indicates that good confinement and high $\beta$ can also be reached with relaxed $q$ profile, possibly for similar reasons. For reaching high $\beta$, the JET-ILW power scans are mainly heated by neutral beam injection (NBI), which tends to produce plasmas with $T_{\mathrm{i}}>T_{\mathrm{e}}$ and simultaneously generates a fast ion population and plasma rotation. All of these physics effects are known to influence turbulent transport. Fortunately, various techniques are available to partially disentangle them experimentally, such as torque balanced NBI at DIIID [13], the use of ion/electron cyclotron resonance heating (ICRH/ECRH) at ASDEX Upgrade [14], or ICRH at JET [15], for example. The WEST tokamak [16] will use ICRH and lower hybrid heating (LH), while JT60-SA [17, 18] will install negative ion NBI and ECRH. However, since the conditions of future devices cannot exactly be matched, it is essential to gain improved understanding also on a theoretical level.
Table 2. Species parameters at $\rho_{\text {tor }}=0.33$.

\begin{tabular}{lllll}
\hline 84798 & $e$ & $D(i)$ & $W$ & $f$ \\
\hline$T_{0} / T_{0 e}$ & 1.000 & 0.852 & 0.852 & 7.889 \\
$n_{0} / n_{0 e}$ & 1.000 & 0.981 & $2.6 \mathrm{E}-5$ & 0.017 \\
$R / L_{T}$ & 4.371 & 3.628 & 3.628 & 4.820 \\
$R / L_{n}$ & 0.750 & 0.657 & 0.294 & 6.327 \\
\hline $84792 q=1.24$ geom & $e$ & $D(i)$ & $W$ & $f$ \\
\hline$T_{0} / T_{0 e}$ & 1.000 & 1.180 & 1.180 & 6.380 \\
$n_{0} / n_{0 e}$ & 1.000 & 0.946 & $4.8 \mathrm{E}-5$ & 0.050 \\
$R / L_{T}$ & 3.968 & 4.582 & 4.582 & 3.863 \\
$R / L_{n}$ & 1.498 & 1.327 & 1.327 & 4.732 \\
\hline $84792 q=0.915$ geom & $e$ & $D(i)$ & $W$ & $f$ \\
\hline$T_{0} / T_{0 e}$ & 1.000 & 1.180 & 1.180 & 6.380 \\
$n_{0} / n_{0 e}$ & 1.000 & 0.946 & $4.8 \mathrm{E}-5$ & 0.050 \\
$R / L_{T}$ & 4.070 & 4.698 & 4.698 & 3.961 \\
$R / L_{n}$ & 1.536 & 1.361 & 1.361 & 4.852 \\
\hline
\end{tabular}

Note: For discharge 84792, mapping to the equilibrium files with $q=1.24$ and $q=0.915$ are shown.

In this paper, we present a detailed gyrokinetic study for two discharges of the low $\delta$ power scan in ILW configuration. Employing the gyrokinetic turbulence code GENE [19], we specifically address turbulence in the inner core, where increased power is observed to yield a steeper ion temperature profile. The computations are performed in realistic geometry, taken from an interpretative CRONOS [20] analysis of experimental data. For the high power case, a $q$ profile sensitivity study is performed. This is important for determining whether the real time control of $q$ is essential for the transition to the advanced regime, or just for having a safe operation. The experimental parameters are summarized in section 2. Details of turbulence modelling are reviewed in section 3, and simulation results are presented in section 4 . Turbulent fluxes are then compared to CRONOS power balance results, whereby turbulence regime transitions are characterized by comparing the results for low and high power discharges. This procedure enables to identify key aspects of turbulence reduction mechanisms at increased power and forms the basis of extrapolation to future tokamaks. Some conclusions are drawn in section 5.

\section{Experimental parameters}

For the low $\delta$ discharges JET84798 $(P=6 \mathrm{MW})$ and JET84792 $(P=13 \mathrm{MW})$, interpretative integrated modelling with the CRONOS suite of codes is performed to self-consistently extract the parameters for thermal and suprathermal plasma species, as well as the magnetic geometry. The selected time window $t=45.2 \mathrm{~s}-45.45 \mathrm{~s}$ is the same as in [4], where these experiments are documented in great detail. For linear and nonlinear gyrokinetic analysis, we focus on the inner core region $\rho_{\text {tor }}=0.33$, where the measured data indicates a steeper ion temperature gradient in the high power discharge. The corresponding parameters from CRONOS are summarized in tables 1 and 2. The main quantity compared to gyrokinetic simulations is the total 
power $\left\langle Q_{j} V^{\prime}\right\rangle$ transported through the $\rho_{\text {tor }}=0.33$ flux surface of the area $V^{\prime}$. Here, $\left\langle Q_{s}\right\rangle$ is the time-averaged turbulent energy flux density for plasma species $j$. The radial coordinate $\rho_{\text {tor }}$ is normalized to $L_{\text {ref }}=\left(\psi_{\text {tor,sep }} / \pi B_{0}\right)^{1 / 2}$ with $\psi_{\text {tor,sep }}$ being the toroidal flux at the separatrix. Thus, the normalized inverse gradient length of a flux-function $A$ is defined as $R / L_{A}=-\hat{R} A^{-1} \mathrm{~d} A / \mathrm{d} \rho_{\text {tor }}$ with $\hat{R}=R / L_{\text {ref. Further, we define }}$ $\rho^{\star}=\rho_{s} / L_{\text {ref }}$ and $\nu_{\mathrm{i}}^{\star}=4 \nu_{i i} q R /\left(3 \pi^{1 / 2} v_{T i} \epsilon^{3 / 2}\right)$, where $\rho_{S}=c_{S} / \Omega_{\mathrm{i}}$ is the reference gyroradius, $c_{s}=\left(T_{0 e} / m_{D}\right)^{1 / 2}$ is the reference (sound-) velocity, $\Omega_{j}=m_{j} c /\left(e B_{0}\right)$ is the Larmor frequency, $\nu_{j j^{\prime}}=4 \pi n_{0 j} q_{j}^{2} q_{j^{\prime}}^{2} \ln \Lambda\left(2 T_{0 j}\right)^{-3 / 2} m_{j}^{-1 / 2}$ is the collision rate for species $j$ of charge $q_{j}$ colliding with species $j^{\prime} v_{T j}=\left(2 T_{0 j} / m_{j}\right)^{1 / 2}$ is the thermal velocity, and $\epsilon=\rho_{\text {tor }} L_{\text {ref }} / R$. The electron beta is defined as $\beta_{\mathrm{e}}=8 \pi n_{\mathrm{e}} T_{\mathrm{e}} / B_{0}^{2}$ and $\alpha=-q^{2} R \sum_{s} \beta_{j}^{\prime}$ is the normalized pressure gradient (with respect to $\rho_{\text {tor }}$ ) summed over all species. The instability threshold for EM modes such as KBM or Alfvénic modes is often approximated by the infinite-n ballooning limit $\alpha_{\text {crit }}$, or $\beta_{\text {crit }}^{\prime}=\alpha_{\text {crit }} / q^{2} R$, respectively, $[21,22]$. While at large magnetic shear $\hat{s}>0.6$, one finds $\alpha_{\text {crit }} \sim 0.6 \hat{s}$, the threshold becomes largely independent of $\hat{s}$ at lower shear, which is in line with our observations in the plasma core. While kinetic corrections are to be expected, this result already points out that the EM threshold is strongly sensitive to $q$ and the total pressure gradient. For this reason, we add an alternative equilibrium (84792 alt.) with lower central safety factor $q=0.915$ ( $\operatorname{similar} \hat{s}$ ) and thus lower $\alpha$ for the high power discharge, which is obtained from the EFIT code constrained by MSE measurements. Realistic values of $q$ may be found in between these limits of $q=1.238$ and $q=0.915$, since the absence of MHD activity (measured by magnetic pickup coils) indicates that $q$ does not drop far below $q=1$ throughout the plasma core.

We note that for both geometry files we have used the same $\rho_{\text {tor }}$ grid from CRONOS for mapping measured profiles and computing gradients, which are then input into GENE. Differences in the gradient parameters and $\beta$ thus origin from the fact that the numerical tracing of the two equilibria [23] yields a slightly different reference length $L_{\text {ref }}$ and magnetic axis field strength $B_{0}$. In a way, this reflects the uncertainties in the simulation parameters in tables 1 and 2 due to equilibrium mapping. Further, no measurement of the $Z_{\text {eff }}$ profile are available for the present discharges. This poses uncertainty to the plasma composition and to the $q$ profile evolution, and stresses the importance of the sensitivity study.

\section{Setup for gyrokinetic simulations}

We use the gyrokinetic code GENE in the flux-tube framework (local in the radial coordinate $x$ ). For ion-scale turbulence, the maximum binormal wavenumbers $k_{y}$ cover is about $k_{y} \rho_{s} \sim 4$, Some electron scale simulations have been performed with $k_{y} \rho_{s}<64$, in order to determine potential contributions to electron transport. The main focus of this work is put on ion-scale turbulence, however, and extremely expensive multiscale simulations have been avoided. The domain size for typical ion-scale turbulence simulations is $l_{x}=240 \rho_{s}, l_{y}=120 \rho_{s}$
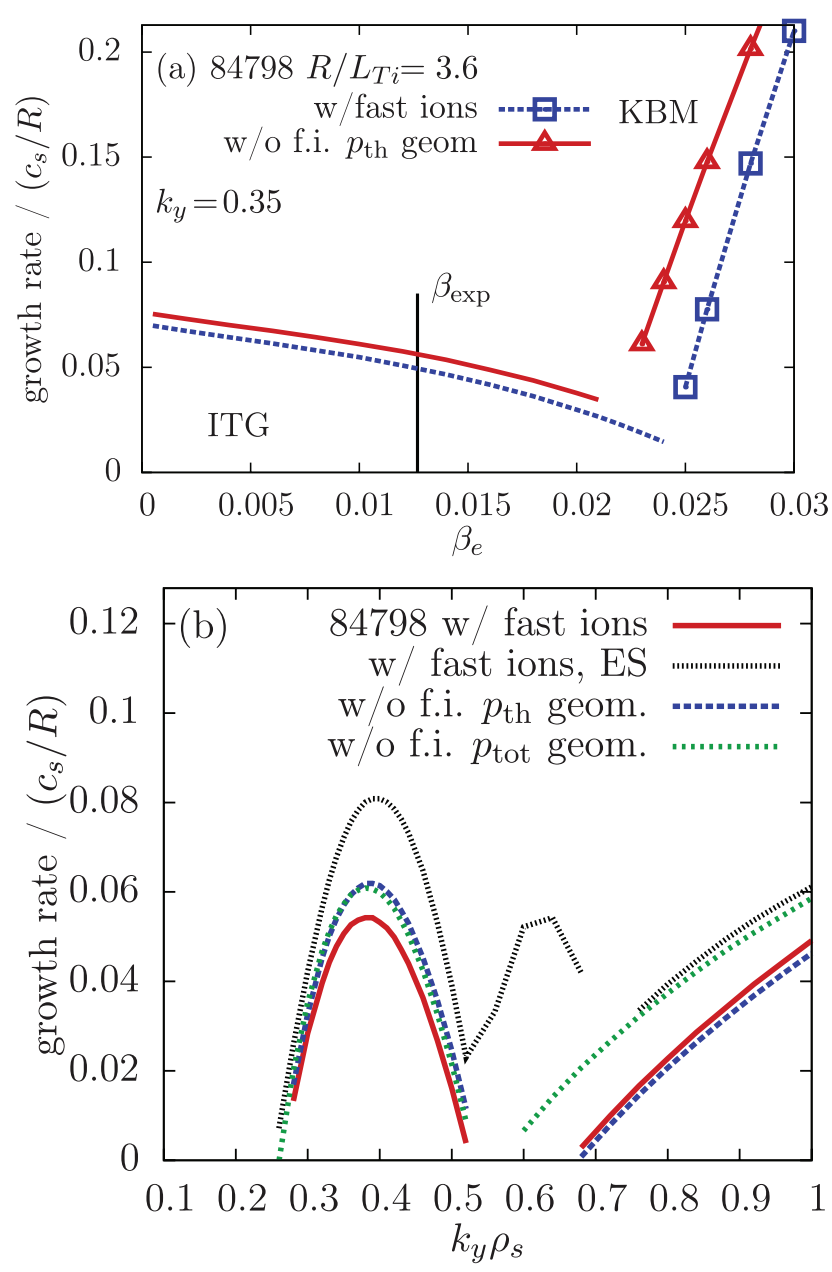

Figure 1. Microinstabilities in the low power discharge 84798: ITG is marked by lines, KBM/BAE by additional symbols. (a) $\beta$ scan at fixed equilibrium (minimum $\beta_{\text {crit }}$ around $k_{y} \sim 0.35$ ), (b) linear growth rate spectra.

and $l_{v \|}=3 v_{T}, l_{\mu}=9 T_{0 e} / B_{0}$ in velocity space, resolved with $n_{x}=192, n_{y}=96, n_{v}=48, n_{\mu}=16$ grid points, respectively. In the parallel direction, 32 grid points are used. For numerical convergence we focused mainly on direct space by varying $l_{x}, l_{y}, n_{x}$ and $n_{y}$ by factors of 1.5 to 2 in nonlinear simulations. We conclude that trends are captured correctly and no qualitative changes of the simulated plasma turbulence are expected. We mention, however, that simulations at very low turbulence levels of $Q_{\mathrm{i}} \lesssim 0.2 Q_{g B}$ (corresponding to $\left\langle Q_{\mathrm{i}} V^{\prime}\right\rangle \lesssim 1 \mathrm{MW}$ for JET84792 at $\rho_{\text {tor }}=0.33$ ) are very sensitive to small changes in the input parameters. Around the experimental flux level of $\left\langle Q_{\mathrm{i}} V^{\prime}\right\rangle \sim 2.85 \mathrm{MW}$, the simulations are far more robust.

Four species are included by default: electrons, thermal deuterium, beam deuterium ions and a tungsten impurity, which is assumed to be fully ionized. In accordance with the relative unimportance of radiation losses in CRONOS analysis and the extremely low tungsten density, tungsten plays virtually no role for microturbulence and is often ignored. Nevertheless, the effective ion charge $Z_{\text {eff }}$ from table 1 is then kept in the Landau-Boltzmann collision operator for the gyrokinetic simulations. A fully electromagnetic (EM) response is considered, including perpendicular and parallel 

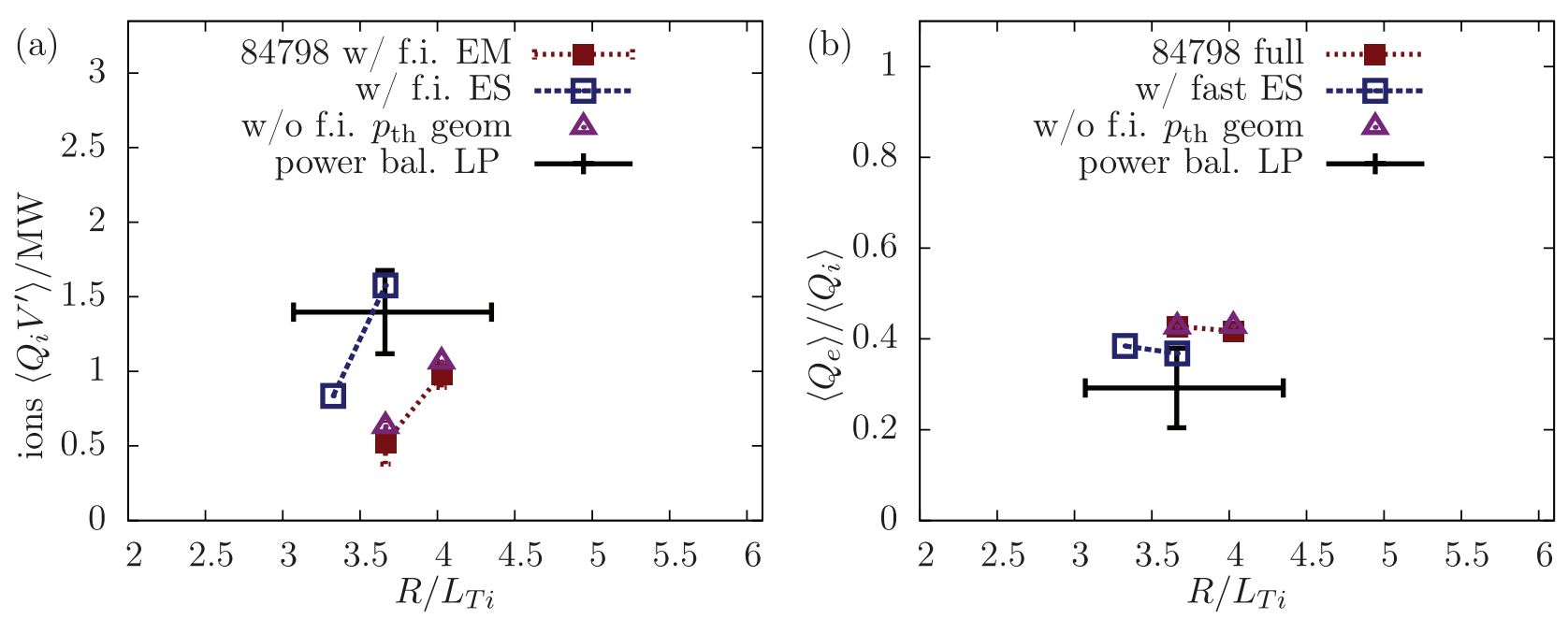

Figure 2. Nonlinear simulations for the low power discharge 84798: (a) ion heat transported through the $\rho=0.33$ surface by ITG turbulence, (b) electron- to ion flux ratio.

magnetic fluctuations. Some runs are performed in the electrostatic (ES) limit by artificially reducing $\beta_{\mathrm{e}}$ to $0.05 \%$, which is essentially equivalent to neglecting $\tilde{A}_{\|}$and $\tilde{B}_{\|}$fluctuations. Employing the $\delta f$ method, the distribution is split into a static Maxwellian part $F_{0}$ and a small, fluctuating part $\tilde{f}$. Fast beam ions are modelled as a fully kinetic species and thus (i) dilute the main ion species, [24], (ii) add to the Shafranov-shift [25], and (iii) dynamically contribute to the $\tilde{\phi}, \tilde{A}_{\|}$and $\tilde{B}_{\|}$fluctuations, $[26,27]$. The latter can have significant impact in nonlinear simulations, [10]. Here, their background distribution is approximated as a Maxwellian with equivalent temperature profile $T_{f}=p_{f} / n_{f}$. The fast ion pressure is accounted for in the Grad-Shafranov solver by default ( $p_{\text {tot }}$ geom). When it is deliberately neglected for dedicated tests, this will be indicated $\left(p_{\text {th }}\right.$ geom). Curvature and $\nabla B$-drifts are computed from this CRONOS magnetic equilibrium in the course of the above mentioned numerical field-line tracing procedure, [23]. Consequently, these quantities remain fixed, when $\beta$ is varied in our simulations. The pressure contribution to the magnetic drifts (see e.g. [28]) is always kept self-consistent with $\beta^{\prime}$, i.e. it changes when species parameters, or $\beta$ are varied. In analytical geometry models, a change in equilibrium pressure would mainly affects the MHD $\alpha$ parameter, which is computed in table 1 from the species parameters (neglecting fast ions yields $\alpha_{\text {th }}$ ). The experimentally determined toroidal rotation profile is accounted for by means of including a parallel flow shear rate $\gamma_{\mathrm{pfs}}$, and a $E \times B$ shearing rate $\hat{\gamma}_{E}=\rho_{\mathrm{tor}} / q \Omega_{\mathrm{tor}}^{\prime}$, [29], which is modelled by $k_{y}$-dependent periodic shifts in $k_{x}$. Furthermore, a GyroLES model for energy transfer to smaller scales determines the magnitude of hyperdissipation in $x, y$ space [30]. In linear simulations, $\gamma_{E}$ and GyroLES are deactivated, but $\gamma_{\mathrm{pfs}}$ is always included.

\section{Simulation results}

Here, we analyze turbulent transport at the radial position $\rho_{\text {tor }}=0.33$ in the two selected discharges 84798 (low power) and 84792 (high power). Besides reconciling power balance heat flux levels with gyrokinetic simulation results, our main goal is to identify physics effects that may lead to increased ion temperature gradient in the high power case. For that reason, we perform nonlinear gyrokinetic simulations. Supportive linear simulations are used to explore the parameter space in more detail.

\subsection{Analysis for the low power $6 \mathrm{MW} N B /$ discharge}

Beginning with the low power discharge, linear simulations show that ion temperature gradient driven (ITG) modes are most unstable. Their growth rate at finite $\beta$ is reduced when compared to the electrostatic limit. The experimental $\beta$ is at about $50 \%$ of the threshold for the onset of Alfvénic modes, which are identified in figure 1(a) by a sharp increase of the growth rate with $\beta_{\mathrm{e}}$ above a critical value $\beta_{\text {crit }}$. This signature is typical for kinetic ballooning mode (KBM) and $\beta$ induced Alfvén eigenmodes (BAE). In our case, the instability is driven by thermal and suprathermal pressure gradients, and its real frequency is close to the one of the geodesic acoustic mode (GAM) - which is expected for BAE. Thus, the label KBM/ BAE is used. The lowest critical $\beta$ for destabilizing KBM/ BAE is found around $k_{y} \rho_{s}=0.35$, the wavenumber chosen in the figure. The full spectrum of figure 1(b) furthermore shows that the impact of fast NBI ions on the geometry is negligible for the ITG branch $\left(0.2<k_{y} \rho_{s}<0.5\right)$, but their contribution as a dynamic species is slightly stabilizing. A trapped electron mode being unstable at $k_{y} \rho_{s}>0.5$ is influenced by the geometry, but, however, does not significantly contribute to the turbulence obtained in nonlinear GENE simulations. In figure 2 the turbulent transport levels are shown to be consistent with the CRONOS power balance analysis around the measured value of the normalized ion gradient $R / L_{T i} \sim 4.2$. The presented nonlinear simulations are restricted to ion scales $\left(k_{y} \rho_{s}<4.8\right)$, but it has been verified in a separate simulation with extended range $k_{y} \rho_{s}<40$ that (for the present parameters) higher-k modes contribute little to heat and particle transport. Thus, at low power, transport is governed by ITG turbulence and is barely influenced by fast ions. However, since $\beta / \beta_{\text {ctit }}$ 

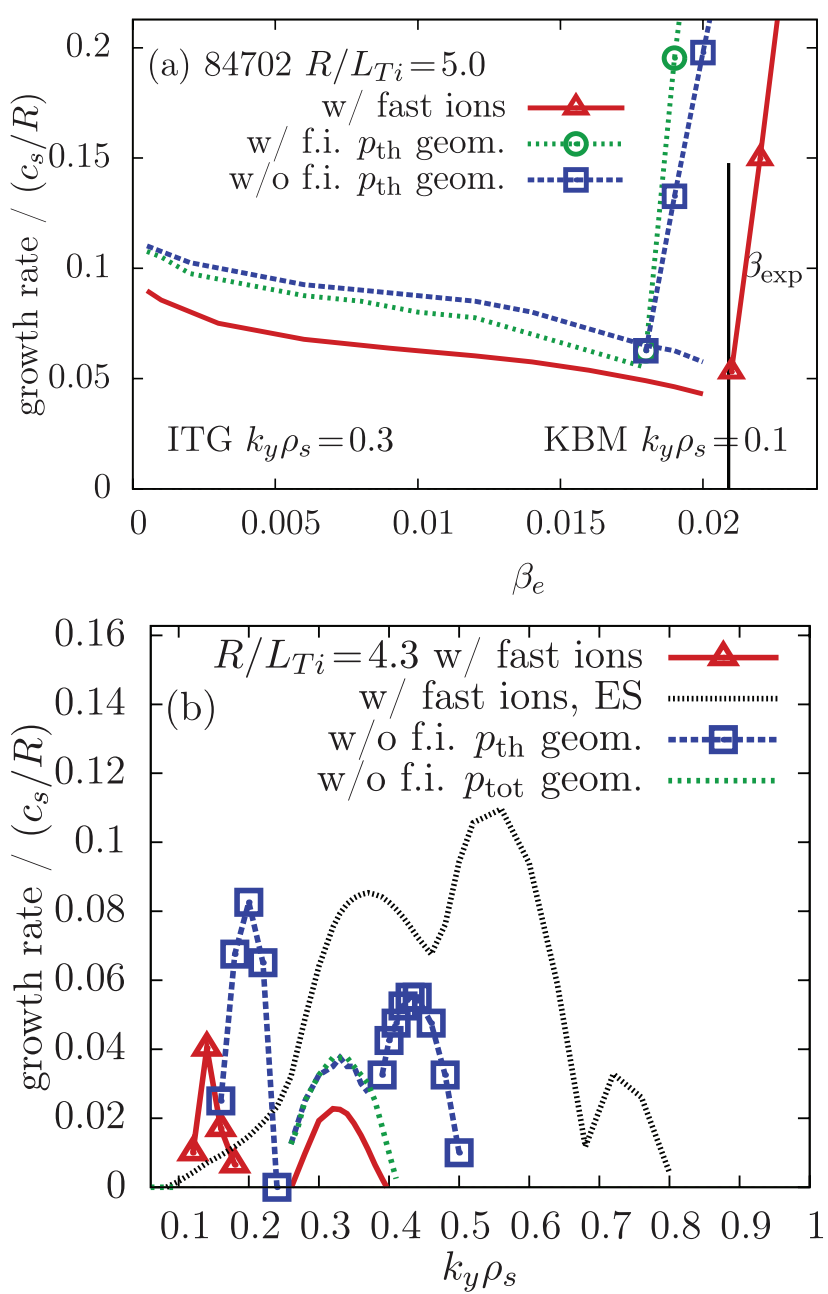

Figure 3. Microinstabilities in the high power discharge 84792. (a) $\beta$-scan at fixed equilibrium (minimum $\beta_{\text {crit }}$ around $k_{y}=0.1$ ), (b) growth rate spectra at $R / L_{T i}=4.3$. ITG modes are marked by lines, $\mathrm{KBM} / \mathrm{BAE}$ by additional symbols.

reaches $50 \%$, EM stabilization already sets in. The procedure of matching the power balance result in an $R / L_{T \text { i }}$ scan yields an up-shift of the temperature gradient by about $20 \%$ from $R / L_{T_{1}}=3.5$ in the ES limit to $R / L_{T \mathrm{i}}=4.2$. A more rigorous analysis of the impact of EM effects on $R / L_{T i}$ would of course have to account for the transport of particles, and momentum.

\subsection{Linear analysis for the high power 13 MW NBI discharge}

At higher power (and thus higher $\beta$ ) EM effects are expected to be more pronounced. Furthermore, the contribution of fast ions is expected to be stronger, due to the increased beam ion density. Indeed, for the high power case, the nominal parameters are very close to the KBM/BAE threshold, as seen in the $\beta$ scan of figure 3(a). For the ITG branch, we show the results for $k_{y} \rho_{s}=0.3$, which is about the last wavenumber stabilized by increased $\beta$. For the KBM branch, $k_{y} \rho_{s}=0.1$ of minimal $\beta_{\text {crit }}$ is chosen. Comparing to the ES result, the maximum ITG growth rate is slightly down-shifted with increased $\beta$. Most linear simulations are performed at $R / L_{T i}=5$, since the turbulence level vanishes below that value (see section 4.3). More details become visible in figure $3(\mathrm{~b})$, where all ion-scale $k_{y}$ wave
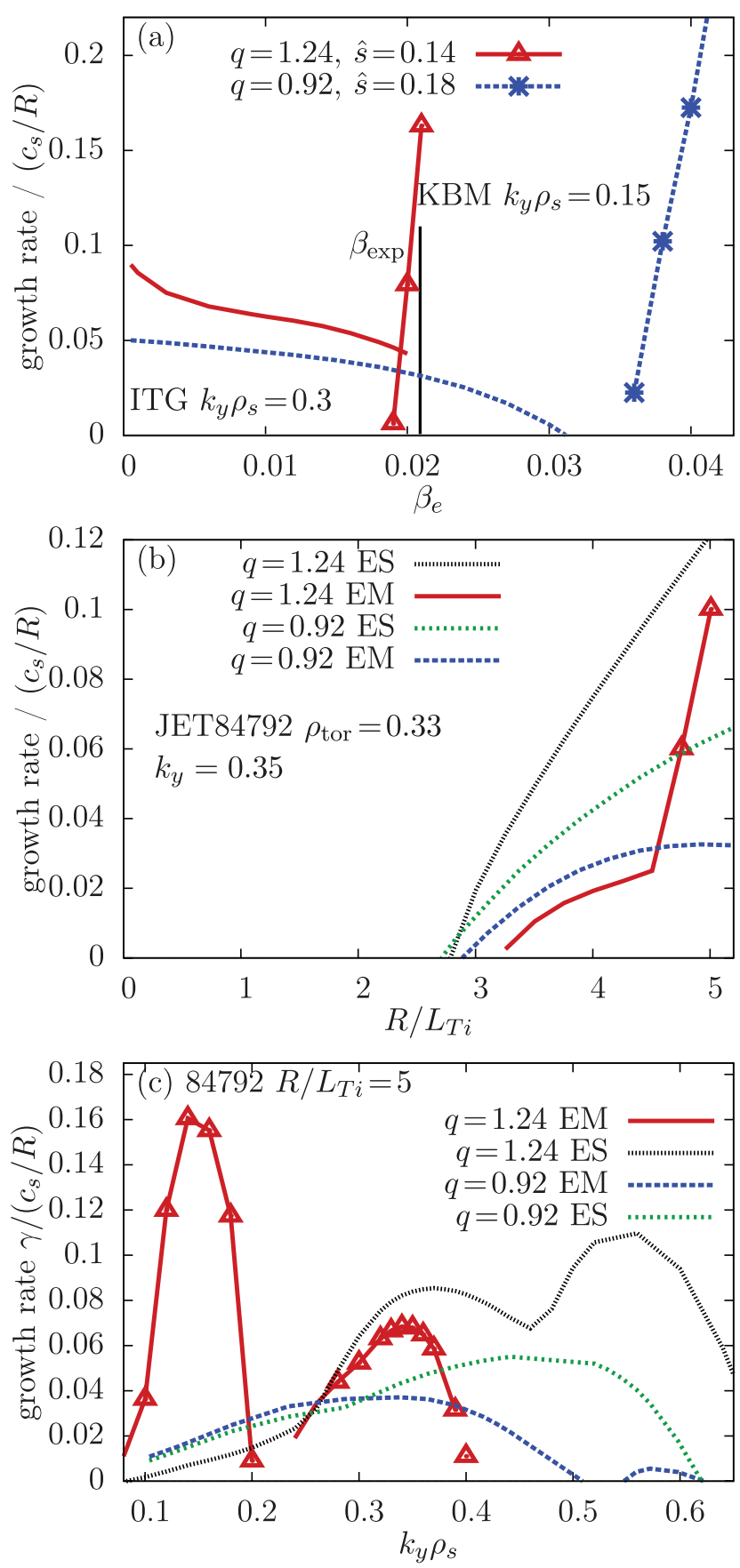

Figure 4. 84792: sensitivity to $q$ profile. (a) $\beta$-scan at fixed equilibria, (b) gradient scan for $k_{y}=0.35$, (c) growth rate spectrum at $R / L_{T i}=5$. ITG modes are marked by lines, KBM/BAE by additional symbols. $\beta_{\text {crit }}$ is larger at lower $q$, the critical gradient is similar and the degree of EM stabilization is much greater with $q=1.24$.

numbers are resolved. As a first observation, the electrostatic limit yields much larger ITG growth rates. In the nominal case, even small variations in $\beta^{\prime}$ change the dominant instability from ITG ( $\operatorname{smaller} \beta^{\prime}$ ) to KBM/BAE (larger $\left.\beta^{\prime}\right)$. We have modified $\beta^{\prime}$ by a $10 \%$ reduction of $\beta$, a $30 \%$ reduction of the fast ion pressure gradient $\nabla p_{f}$, or a change of the thermal pressure gradient (not all are shown). The role of fast ions is twofold: the contribution of the fast ion pressure gradient to $\beta^{\prime}$ dynamically destabilizes KBM/BAE (and stabilizes ITG), while the fast ion 
Table 3. Critical gradient for the two $q$ profiles.

\begin{tabular}{lllll}
\hline$\hat{s}$ & $q$ & Equation (1) & $R / L_{T i, \text { crit,ES }}$ & $R / L_{T i, \text { crit,EM }}$ \\
\hline 0.175 & 0.915 & 3.7 & 2.75 & 2.9 \\
0.145 & 1.24 & 3.38 & 2.75 & 3.25 \\
\hline
\end{tabular}

Note: EM and ES gyrokinetic results use $k_{y}=0.35$, which yields minimum $R / L_{T, \text { crit }}$ for $k_{y} \in\{0.35,0.3,0.25,0.2\}$.

pressure contribution to the equilibrium provides some stabilization of the KBM/BAE branch. Although KBM growthrates increase quickly, once the threshold is overcome, we confirm earlier observations that geometric stabilization (for example due to fast ion pressure) only shifts $\beta_{\text {crit }}$ by $5 \%$ to $10 \%$, [6, 8$]$. In order to study these fast ion effects on ITG, $R / L_{T i}=4.3$ is taken in figure 3(b). At $R / L_{T i}=5$, figure 4(c) shows that most parts of the spectrum are dominated by EM modes instead. For clarification, we note that due to the strong EM stabilization at nominal parameters, the parallel flow shear drive is essential to destabilize the mode that has been labeled as ITG, for simplicity. In a less effective manner, the parallel flow shear also drives the KBM/BAE branch.

For assessing the influence of the $q$ profile on microinstabilities, the alternative CRONOS equilibrium with reduced $q_{\min }$ (and slightly increased $\hat{s}$ ) is used (see table 1 ). Figure 4 shows that the KBM threshold is very sensitive, since $\alpha \propto q^{2}$ is the relevant parameter. In fact, the $\mathrm{KBM} / \mathrm{BAE}$ growth rates are on top of each other, when plotting against $\alpha$. Because $\hat{s}$ is very small, the slight change in $\hat{s}$ does not matter for the value of $\beta_{\text {crit }}$. Also the ITG branch is affected by the equilibrium change. In [31] a formula for the gradient threshold

$$
R / L_{T i, \text { crit }}=\left(1+\frac{T_{\mathrm{i}}}{T_{\mathrm{e}}}\right)\left(1.33+1.91 \frac{\hat{s}}{q}\right) \times \mathcal{G}
$$

is given, which is based on ES gyrokinetic simulations with adiabatic electrons. For our JET cases at $\rho_{\text {tor }}=0.33$, the geometric factor $\mathcal{G}=(1+0.3 \epsilon(\partial \kappa / \partial \epsilon)) \sim 0.998$ does not play a role. As the authors of [31] assume, our low- $q$ low- $\hat{s}$ JET parameters lie outside the applicability regime. In table 3 we find $R / L_{T \text { crit }}$ to be slightly lower than equation (1), with reversed trend in terms of $\hat{s} / q$. However, our results are consistent with the observation of increased gradient threshold at larger $\beta / \beta_{\text {crit }}$, [9]. Probably more importantly, the linear critical gradient is found well below the experimental gradient (and the nonlinear thresholds). It is thus essential that in the $q=0.915$ case, the growth rate is less sensitive to $R / L_{T i}$ at the same wavenumber $k_{y}=0.35$. Furthermore, the instability covers a broader range in $k_{y}$, as shown in figure 4(c).

A notable difference between the high and low power cases is given by the increased temperature ratio $T_{\mathrm{i}} / T_{\mathrm{e}}$ at increased NBI heating. Large $T_{\mathrm{i}} / T_{\mathrm{e}}$ is well known to stabilize ITG turbulence quite efficiently. This can be inferred from equation (1) and is also seen in the linear simulations of figure 5. Recent experimental results confirm this effect in a regime of lower $\beta$, [32]. Increased $T_{\mathrm{i}}$ also contributes to the total pressure and thus KBM modes are destabilized at $T_{\mathrm{i}}=1.4$ above the nominal value. Interestingly, when observing the maximum growth rate, $T_{\mathrm{i}} / T_{\mathrm{e}}$ stabilization is stronger for the $q \sim 1.24$ equilibria
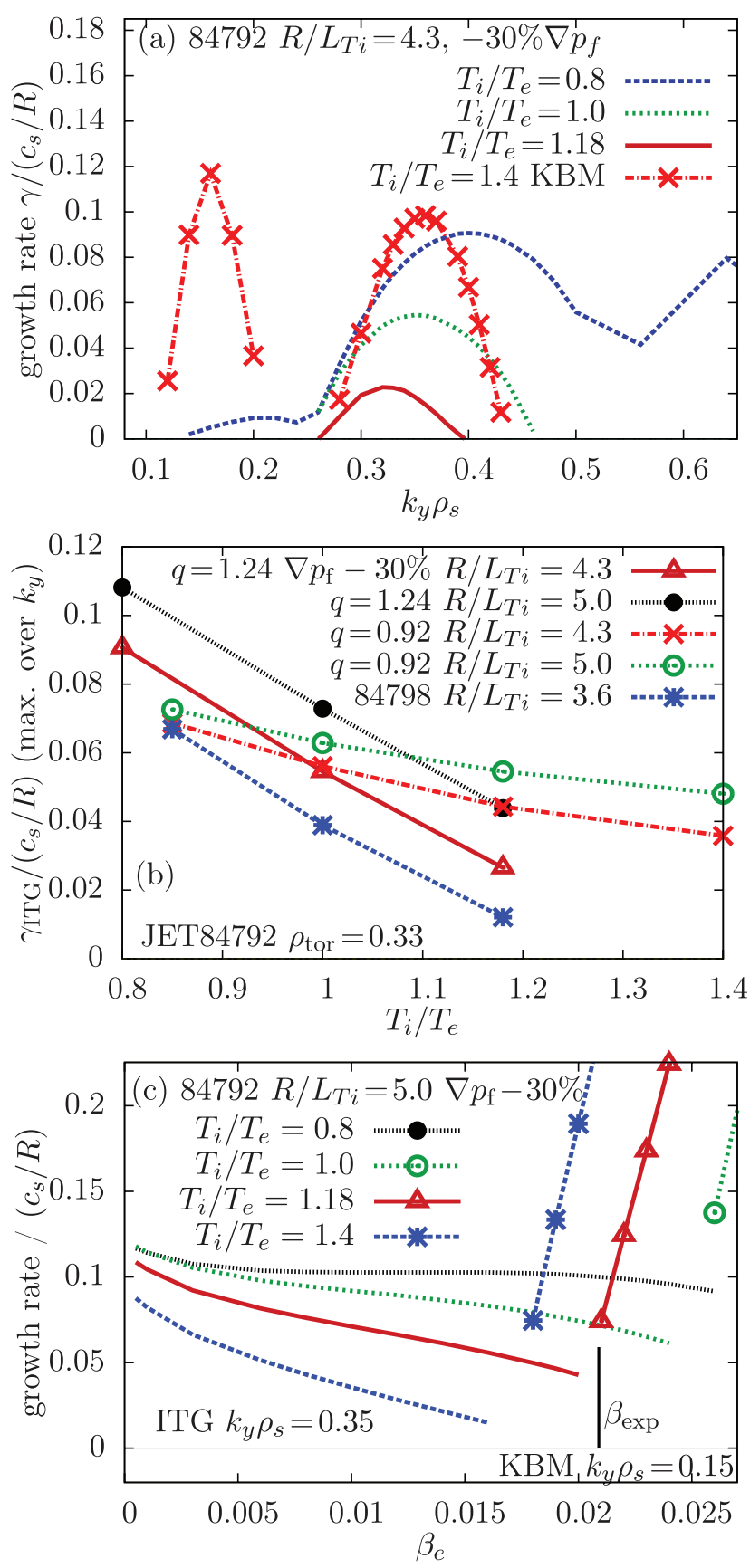

Figure 5. (a) $T_{\mathrm{i}}$ scan at fixed $T_{\mathrm{e}}$ (and $\beta_{\mathrm{e}}$ ) leads to reduced ITG growth rates up to KBM/BAE instability. (b) This effect is stronger in the $q=1.24$ equilibria for both low and high power (only ITG shown). (c) Consistently, $\beta$-stabilization is less effective at smaller $T_{\mathrm{i}}$.

(both at high and low power) as compared to the $q=0.915$ equilibrium. From equation (1) one would expect the opposite, but EM effects appear to be decisive. Indeed, figure 5(c) reveals that the strength of EM stabilization dramatically reduces with decreasing $T_{\mathrm{i}} / T_{\mathrm{e}}$ (at fixed $T_{\mathrm{e}}$ and $\beta_{\mathrm{e}}$ ), as the KBM/ BAE threshold is pushed further away. Note, however, that $\beta_{\text {crit }}$ appears to scale with $\beta_{\mathrm{i}}=T_{\mathrm{i}} / T_{\mathrm{e}} \beta_{\mathrm{e}}$, but the ITG stabilization is not that easily parameterized. Since EM effects are weaker in the $q=0.915$ scenario, $T_{\mathrm{i}} / T_{\mathrm{e}}$ stabilization is thus consistently less effective also. In figure $5(\mathrm{c})$, reduced $\nabla p_{f}$ is used, which 

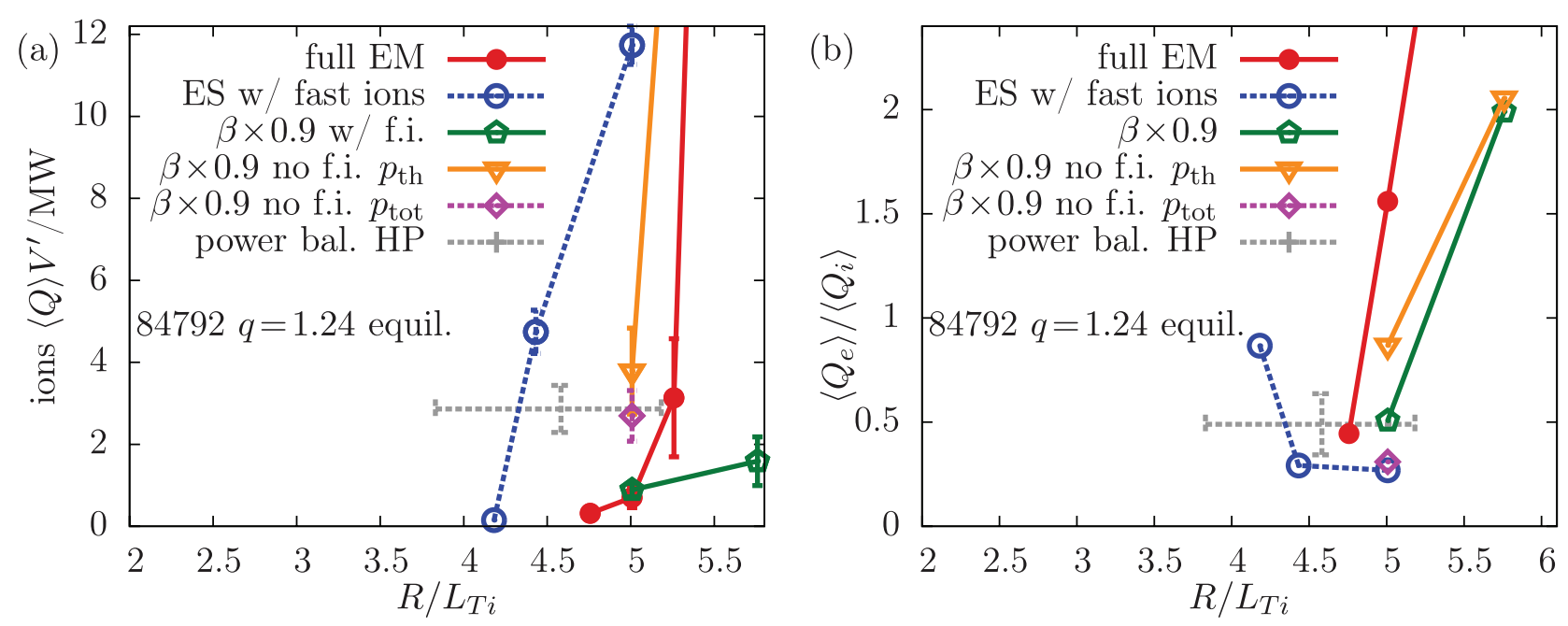

Figure 6. Nonlinear simulations for the high power discharge 84792 with $q=1.24$ equilibrium: (a) turbulent ion heat transport in the ITG and $\mathrm{KBM} / \mathrm{BAE}$ regimes (all with $E \times B$ ). (b) electron- to ion flux ratio.

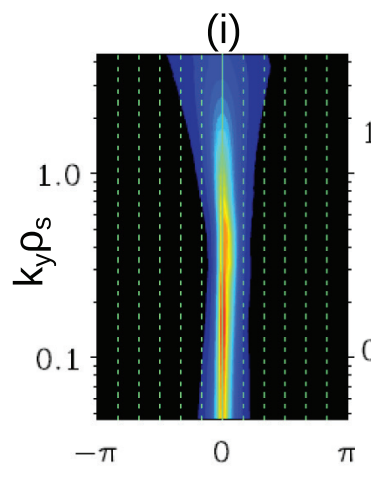

(i)

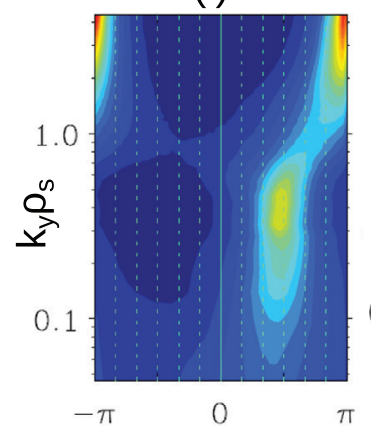

(ii)

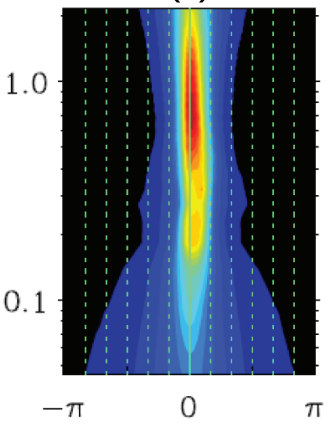

(ii)

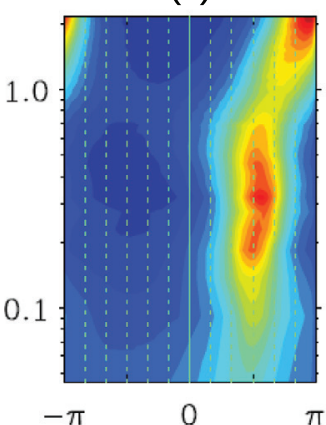

(iii)

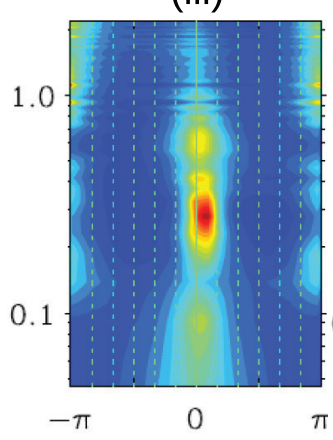

(iii)

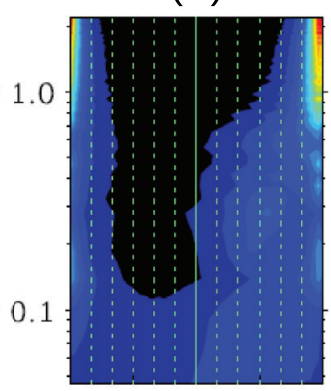

(iv)
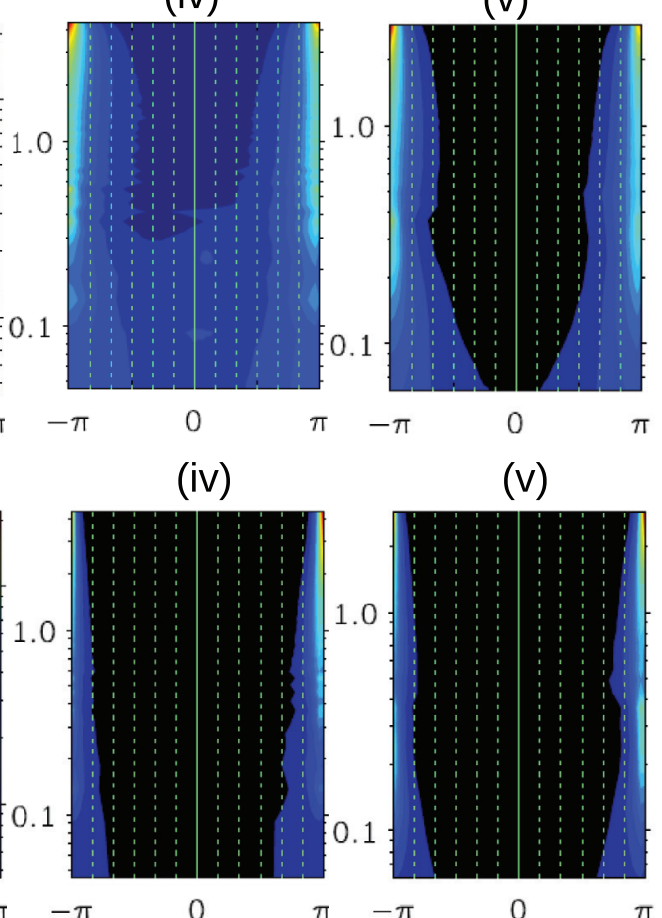

(v)

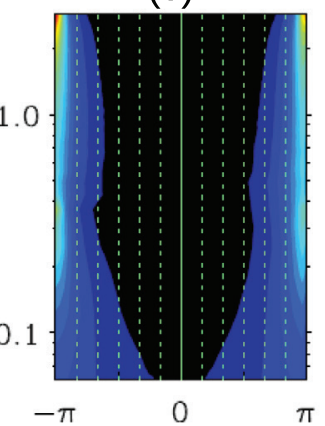

(v)

Figure 7. Histograms of $\tilde{\phi} \times \tilde{n}$ cross phase angle for selected discharges with increasing $\beta / \beta_{\text {crit }}$ from left to right: (i) ES $q=1.24$ geom, $R / L_{T i}=5$. (ii) $\mathrm{EM} q=0.915$ geom. $R / L_{T i}=6$. (iii) $\mathrm{EM} q=1.24$ geom, $\beta \times 0.9, R / L_{T i}=5$. (iv) $\mathrm{EM} q=1.24$ geom, $\beta \times 0.9, R / L_{T i}=5.5$. (v) $\operatorname{EM} q=1.24$ geom, $R / L_{T i}=5.2$. (i) $\alpha\left(\phi \times n_{1 i}\right)$. (ii) $\alpha\left(\phi \times n_{1 i}\right)$. (iii) $\alpha\left(\phi \times n_{1 i}\right)$. (iv) $\alpha\left(\phi \times n_{1 i}\right)$. (v) $\alpha\left(\phi \times n_{1 i}\right)$. (i) $\alpha\left(\phi \times p_{1 i}\right)$. (ii) $\alpha\left(\phi \times p_{1 i}\right)$. (iii) $\alpha\left(\phi \times p_{1 i}\right)$. (iv) $\alpha\left(\phi \times p_{1 i}\right)$. (v) $\alpha\left(\phi \times p_{1 i}\right)$.

moderately increases the KBM/BAE threshold with respect to the full $\nabla p_{f}$ case, but does not generally affect the physics. Besides $\alpha$, also the normalized collision rate $\nu_{\mathrm{i}}^{\star}$ and the flow shear rate $\gamma_{E}$ are sensitive to changes of $q$. While collisions are weak in both cases, the impact of flow shear is expected to be slightly larger in the low- $q$ scenario. This is subject of section 4.3, where nonlinear simulation results are presented.

\subsection{Nonlinear analysis for the high power 13 MW NBI discharge}

Combining our insights from the linear analysis is very helpful to interpret nonlinear simulations for the high-power case, which are presented in the following. Turbulent heat fluxes from GENE are depicted in figure 6 for the $q=1.24$ equilibrium, whereby $R / L_{T i}$ is varied. The electrostatic limit agrees 
with power balance heat flux at $R / L_{T i} \sim 4$.1, which is slightly larger than the gradient in the low-power scenario. This can be attributed to increased $T_{\mathrm{i}} / T_{\mathrm{e}}$, geometric $\alpha$ stabilization and slightly increased $E \times B$ shear. Adopting the more realistic electromagnetic model at nominal $\beta_{\mathrm{e}}$ an even greater up-shift of the nonlinear critical gradient is found, but turbulence has transitioned from the ITG regime to the KBM/BAE regime (the red dots in figure 6). For vizualizing this transition, it is useful to monitor the cross phase angle distribution between two fluctuating quantities, such as $\tilde{\phi}$ and $\tilde{n}$, as done in figure 7 for selected nonlinear simulations. After a Fourier trasform to $\left\{x, k_{y}\right\}$ space, the phase is computed as $\operatorname{Im}\left(\ln \left(\tilde{\phi}_{k_{y}} / \tilde{n}_{k_{y}}\right)\right)$ for each point in $\{x, z\}$ and collected in 62 bins between $-\pi$ and $\pi$. The result is time averaged over the saturated turbulence. For ITG, the dominant $\tilde{\phi} \times \tilde{n}_{\text {i }}$ phase is close to zero, while it is close to $\pi$ for the KBM/BAE. Notably, this is different from gyrofluid ideal ballooning turbulence, from which a cross phase of $\pi / 2$ is expected, [33]. Together with the relatively larger electron thermal transport $Q_{\mathrm{e}}$ (with a strong magnetic flutter component), this observation points towards a stronger role of electron dynamics in the KBM/BAE case. For the present parameters, these $\mathrm{KBM} / \mathrm{BAE}$ simulations are stable in time and develop a regular turbulent spectrum. For other setups, a runaway-phenomenon (see e.g. [34, 35]) has been found, or turbulence develops a sharp peak about a single binormal wavenumber, [36]. However, when the ion flux is matched at $R / L_{T i} \sim 5.2$, the electron transport levels are 5-10 times larger than power balance, and the fast particle transport is also very large. Assuming a quasi-stationary state, in which heat sources are balanced by turbulent transport at constant background profiles, the KBM regime is thus inconsistent with the experiment in terms of $Q_{\mathrm{e}} / Q_{\mathrm{i}}$.

Reducing $\beta$ by $10 \%$ brings us back to an ITG turbulence regime at $R / L_{T i}=5$. This ITG regime is difficult to resolve numerically, because the turbulence level is very low. At higher $R / L_{T i}$ the ITG gradually transitions to $\mathrm{KBM} / \mathrm{BAE}$, as monitored by a $\phi \times n_{1}$ cross phase shift from zero to $\pi$ in figure 7 (iii) $\rightarrow$ (iv) and the increase in $Q_{\mathrm{e}}$ above the experimental level. Thus, it is difficult to match power balance heat fluxes at $q=1.24$. The impact of fast ions is nevertheless investigated in this $10 \%$ reduced $\beta$ setup, since it allows to access the EM stabilized ITG turbulence regime. Nonlinear simulation results are collected in table 4. Already from the linear simulations of figure 3 we expect to find a KBM-type regime when fast ions are neglected in dynamics and equilibrium pressure, even at $10 \%$ reduced $\beta$. Indeed, at $R / L_{T i}=5$, the heat flux is around the experimental value with strong increase at higher gradients, KBM-like cross phase and large $Q_{\mathrm{e}} / Q_{\mathrm{i}}$. Removing fast ions only from the dynamics, but not from the equilibrium yields approximately the same ion heat flux around $R / L_{T i}=5$, but turbulence is of ITG character. Comparing this to the very low turbulence level in case of fully included fast ions, the reduction of ITG turbulence due to a dynamic fast ion species is confirmed by nonlinear simulations. In all finite- $\beta$ simulations in the $q=1.24$ case, we find similarly to [6] that switching off $E \times B$ flow shear has a very little effect on the turbulence level and thus on the gradient
Table 4. Impact of fast ions in nonlinear simulations with $q=1.24$ equilibrium (with $E \times B$ ).

\begin{tabular}{lllll}
\hline $84792, q=1.24, R / L_{T i}=5$ & $\left\langle Q_{\mathrm{i}} V^{\prime}\right\rangle$ & $Q_{\mathrm{e}} / Q_{\mathrm{i}}$ & $\phi \times n_{i 1}$ & Type \\
\hline$\beta \times 0.9$ no f.i. $p_{\text {th }}$ geom & $3.79 \mathrm{MW}$ & 0.87 & $\pi$ & KBM \\
$\beta \times 0.9$ no f.i. $p_{\text {tot }}$ geom & $2.70 \mathrm{MW}$ & 0.31 & 0 & ITG \\
$\beta \times 0.9$ w/ f.i. $p_{\text {tot }}$ geom & $0.89 \mathrm{MW}$ & 0.51 & 0 & ITG \\
CRONOS & $2.86 \mathrm{MW}$ & 0.49 & & \\
\hline
\end{tabular}

Note: ITG is stabilized by dynamic fast ions in $p_{\text {tot }}$ geometry. With $p_{\text {th }}$ geometry this is masked by the transition to KBM/BAE turbulence.

threshold (not shown). In fact, $E \times B$ flow shear can increase transport, especially the magnetic flutter component.

Turning now to the $q=0.915$ geometry, we have seen $\beta_{\text {crit }}$ to be significantly increased. Indeed, here we observe ITG turbulence further away from the KBM regime, but still of electromagnetic character, which matches the experimental flux at a relatively high $R / L_{T i} \sim 5.5$. A fascinating interplay between the turbulence reduction due to $\beta$ effects and due to rotation $(E \times B$ shear) is observed in figure 8: while the ES simulations without $E \times B$ predict $R / L_{T i} \sim 4.3$, switching on EM effects at no $E \times B$ shear yields a significant up-shift to $R / L_{T i} \sim 5.2$. Adding $E \times B$ shear to this EM simulation yields a further small up-shifts to $R / L_{T i} \sim 5.5$. Interestingly, the ES simulation with $E \times B$ even exhibits a $7 \%$ higher gradient threshold $R / L_{T i} \sim 5.9$ than the full-physics case (EM with $E \times B)$.

This has important ramifications for widely used simplified (e.g. quasi-linear) models: an electrostatic approach with $E \times B$ shear may succeed to describe the present JET plasmas, just as a sophisticated electromagnetic model (correctly mimicking nonlinear $\beta$-stabilization) does. However, this coincidence with the experiment would occur for the wrong reason: The efficiency of $E \times B$ suppression in ES simulations can mask the EM character of the system. Extrapolations will thus fail for machines like ITER, where rotation is low, but $\beta$ can still be high.

For improving on simplified models it is instructive to analyze the turbulence spectra of $|\phi|^{2}$ and $Q_{\mathrm{i}}$ in figure 9 at constant gradient $R / L_{T i}=6$. In the ES case, $E \times B$ shear acts as expected: the low- $k$ part of the spectra drops, so that the region of strongest transport shifts towards higher $k_{x}$ and $k_{y}$. In simulation without $E \times B$ shear, EM effects shift the spectra to lower $k_{x}$ and $k_{y}$. Adding $E \times B$ in the EM case shifts the spectra to higher $k$ (as in the ES case), but the drop of transport is much less pronounced. The total energy flux $Q_{\mathrm{i}}$ even rises (within the standard deviation of the fluctuating time traces). In figure 9(b) $\phi$ amplitudes are largest in the $\mathrm{EM}+E \times B$ case, because a strong zonal (flux-surface averaged) component develops, which does not directly impact on the transport. One possible reason for $E \times B$ being less effective in strongly EM cases is that the turbulence gains more of an Alfvenic character and thus develops faster time scales. From Fourier transforming the $\phi$ fluctuations in time, we indeed find that the dominant EM frequency at $k_{y}=0.32$ is $0.185 c_{s} / L_{\text {ref }}$, which is $25 \%$ larger than the dominant ES frequency. Furthermore, the EM linear mode structure is known to develop finer radial scales which are less efficiently sheared apart. 

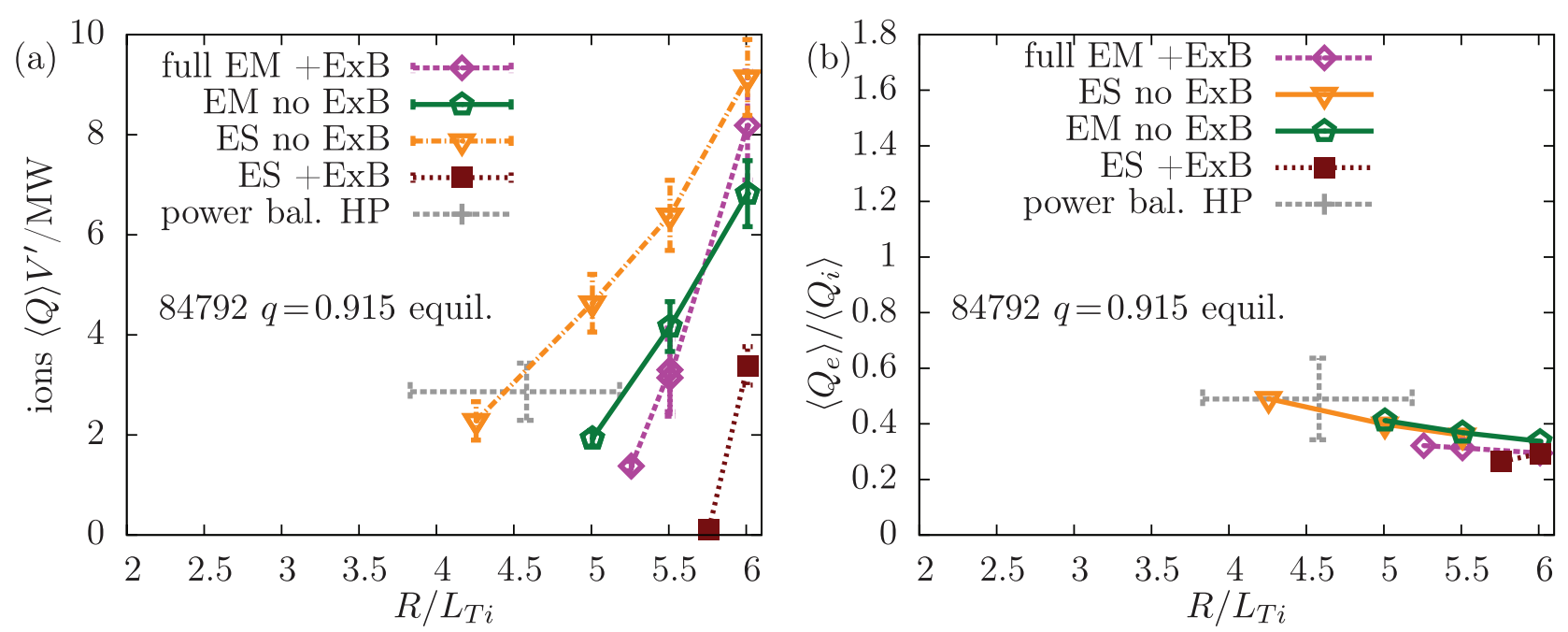

Figure 8. Nonlinear simulations for the high power discharge 84792 with $q=0.91$ equilibrium: the role of EM and $E \times B$ stabilization.
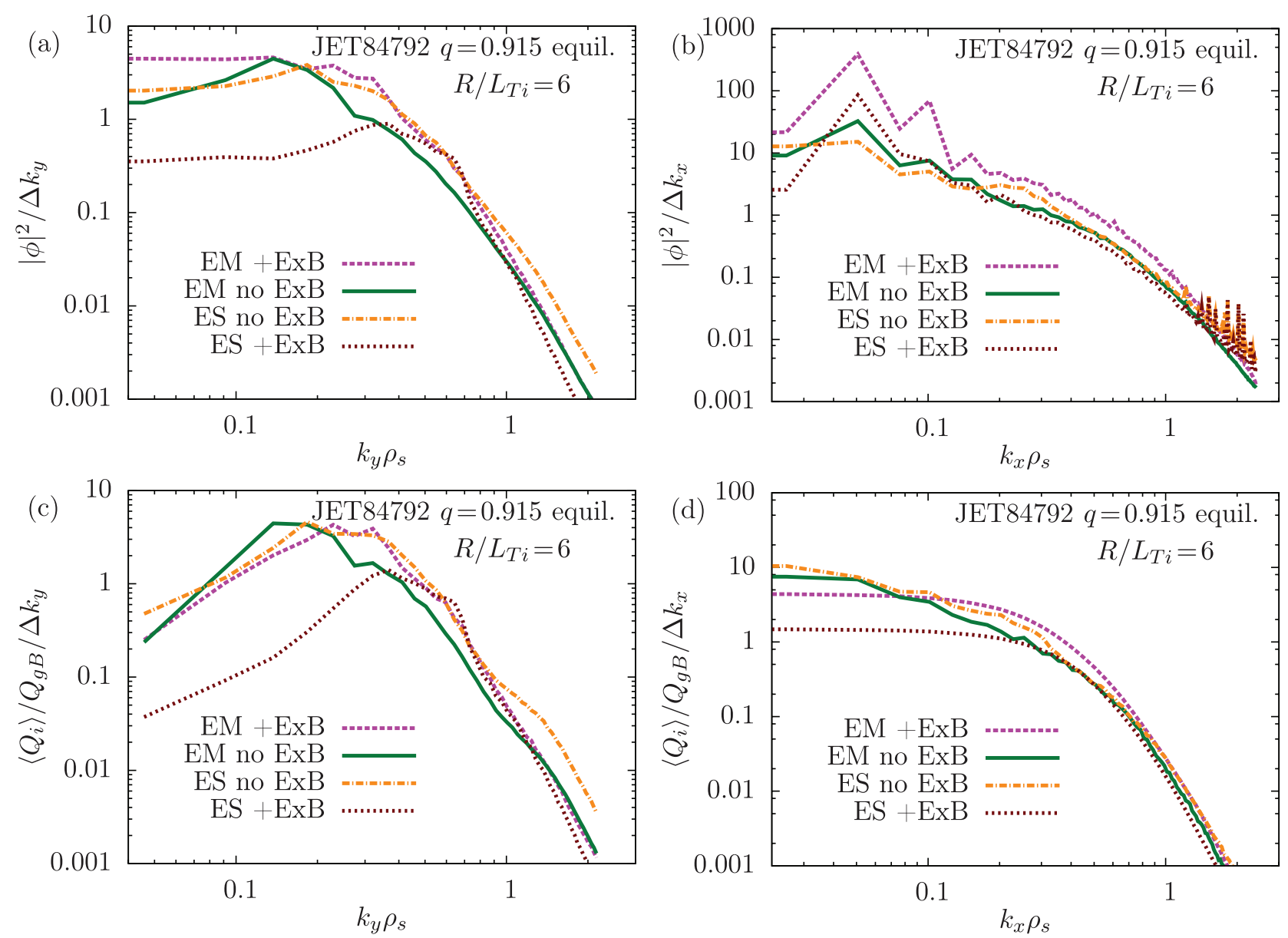

Figure 9. Impact of $\beta$ and $E \times B$ effects on turbulence spectra of the high power discharge 84792 with $q=0.915$ equilibrium at constant gradient $R / L_{T i}=6$, where all combinations exhibit a finite turbulence level.

\subsection{Discussion of q profile sensitivity}

For the $q=1.24$ equilibrium, one finds either KBM turbulence, which yields $Q_{\mathrm{e}} / Q_{\mathrm{i}}$ inconsistent with the experiment, or ITG turbulence (at reduced $\beta$ ), which yields a very low turbulence level. We note that in such cases of marginal ITG stability and sufficient electron temperature gradient (ETG) drive at small-scales, cross-scale-coupling has been identified to hinder the generation of zonal flows, [37, 38], and thus potentially increases the ion-scale ITG transport by some factors. However, in the process of varying the equilibrium by changing mainly the $q$ profile, the ITG turbulence regime is found to be further away from marginality. Here, ETG turbulence may contribute some fraction of the electron flux [39] but is not expected to strongly react back to ion scales. In this 
$q=0.915$ equilibrium, the experimental fluxes are successfully recovered at increased $R / L_{T i}$.

The two investigated cases can be considered as upper and lower limits, between which realistic $q$ profiles can exist. This is because the absence of signatures for large-scale MHD modes in the experimental diagnostics indicates that $\mathrm{q}$ does not drop significantly below $q=1$ throughout the plasma core. On the other hand, $q>1.24$ appears to favour KBM turbulence too strongly, which is excluded by the large $Q_{\mathrm{e}} / Q_{\mathrm{i}}$ flux-ratio. Assuming the intermediate value of $q=1.1$, for example, we obtain $\beta / \beta_{\text {crit }} \sim 0.74$ from the simple $q^{2}$ scaling found in our linear simulations. Having in mind that the low power discharge (at $q=1.23$ ) was already affected at $\beta / \beta_{\text {crit }} \sim 0.5$, this estimate suggests that EM stabilization (supported by dynamic fast-ions) contributes significantly to an increased ion temperature gradient, and thus to the beneficial confinement scaling. For the low power discharge, no $q$ profile sensitivity study has been performed, because changes are expected to be less prominent for two reasons: (i) the original parameters at $q=1.24$ are already quite far from $\beta_{\text {crit }}$ and (ii) the impact of $E \times B$ shear, which could mask EM stabilization, is smaller due to lower rotation.

\section{Summary and conclusions}

We have performed gyrokinetic simulations for two JET-ILW discharges of nearly identical (broad) $q$ profile, but a factor-of-two difference in absorbed power. By direct comparison we were able to identify some of the key physics effects that are believed to contribute to the relatively good $\tau_{E} \sim P^{-0.3}$ power-scaling behavior of the experimental power scan. In the inner core $\rho_{\text {tor }}=0.33$, gyrokinetic simulations matched CRONOS power balance at values of $R / L_{T i}$ that are significantly up-shifted at high power, which corresponds to a steeper ion temperature profile. Since turbulent transport increases rapidly above the gradient threshold (profile stiffness), the steeper gradient can not be attributed to the larger source alone. Improved core confinement rather origins from the change of plasma parameters. In both discharges we found ITG turbulence to be the best candidate for explaining the experimentally determined heat fluxes. Finite $\beta$ effects begin to reduce the turbulence level already at low power. At high power, we have studied a set of two $q$ profiles, which can be viewed as upper and lower limit within the measurement uncertainties. The first one $(q=1.24)$ yields increased $R / L_{T i}$ and is close to the transition between strongly EM-stabilized ITG turbulence and KBM/BAE turbulence, the latter being excluded by its high electron thermal transport level. In the second equilibrium $(q=0.915)$, ITG turbulence of less electromagnetic character confirms the experimentally determined gradient up-shift. The absence of large-scale MHD modes indicates that $q$ does not drop much further below one, though. Also intermediate $q$ profiles are supported by our simulations: assuming $q=1.1$, for example, an ITG turbulence regime is expected to yield significantly increased $R / L_{T i}$ with respect to the low-power discharge.
This $R / L_{T i}$ up-shift has been identified to origin from an interplay between multiple effects: Larger dynamic EM stabilization due to higher (thermal and fast ion) $\beta$, increased thermal and suprathermal equilibrium pressure (Shafranovshift), and stabilization due to larger $T_{\mathrm{i}} / T_{\mathrm{e}}$. Finally, $E \times B$ flow shear suppression may be non-negligible as well, but the associated gradient up-shift is expected to be $10 \%$ (found for fully $\mathrm{EM} q=0.915$ case) or smaller. With respect to the interplay of rotation effects with electromagnetic physics, we confirm earlier $\mathrm{C}$-wall results that $E \times B$ flow shear suppression becomes less relevant, the more electromagnetic the system is. In this context, it is important to note that for the $q=0.915$ geometry, the impact of $E \times B$ flow shear yields a very large $R / L_{T i}$ up-shift in the electrostatic limit. This can mask the importance of finite- $\beta$ effects and has important ramifications for widely used simplified (e.g. quasilinear) models: While electrostatic models with $E \times B$ shear may succeed for the current JET plasmas, their extrapolations to low-rotation devices like ITER will fail, since $\beta$ can still be high.

Concerning $q$-profile optimization in present machines, we conclude from our simulations that elevated $q$ at low shear generates strong electromagnetic stabilization of ITG turbulence, which could allow steeper gradients. On the other hand, above the KBM/BAE limit, strong transport is expected. However, KBM/BAE turbulence yields a larger electron to ion flux-ratio than CRONOS interpretative power balance, from which we conclude that the KBM/BAE threshold is not surpassed in the present experiments. It is not entirely clear, whether higher $q$ would lead to the undesirable instability of large-scale MHD ballooning modes, or if rather the plasma profiles will be limited by turbulence before a disruption takes place. Previous gyrokinetic results indicate that the turbulence threshold is indeed lower than the ideal ballooning limit. If those turn out to be correct in the present regime, interesting experiments could attempt to elevate the $q$ profile, while maintaining low magnetic shear. Taking the present high power low $q$ case as a starting point, we have identified the $E \times B$ shear effect to only contribute at most $10 \%$ to the gradient upshift, which may not be decisive, at least in the inner plasma core. When the system becomes even more electromagnetic at larger $q$, the stabilizing impact of $E \times B$ shear decreases further, or even turns to enhancing transport. Disentangling rotation and electromagnetic effects for better prediction highperformance plasmas can be subject of dedicated experiments. Low rotation can be obtained at DIII-D with balanced NBI, at ASDEX Upgrade and JET by mixing in ICRH heating, or at WEST (ICRH $+\mathrm{LH})$, provided that the installed heating is sufficient to reach the required high $\beta$ values.

We now turn to discussing the relevance and the implications of our results for future devices, like ITER. Since the heating methods apply much less torque to the plasma, $E \times B$ shear is expected to be much lower in ITER. While this lack of $E \times B$ suppression is expected to yield larger turbulence levels in electrostatic systems, no big effect is expected in strongly electromagnetic cases found in the inner core of JET 'hybrid' discharges. In contrast, thermal EM stabilization is expected to directly transfer, provided that $\beta_{\text {crit }}$ is closely approached. However, the ratio $T_{\mathrm{i}} / T_{\mathrm{e}}$ scales less favourably, because ITER 
will dominantly generate electron heating (by fusion-born $\alpha$ particles, for example) and thus likely operate at $T_{\mathrm{e}} \gtrsim T_{\mathrm{i}}$. Linear simulations have revealed an interplay between $\beta_{\mathrm{e}}$ and temperature ratio, whereby EM stabilization is less effective for $T_{\mathrm{e}}>T_{\mathrm{i}}$. These findings indicate that it may be more complicated to access highly EM-stabilized regimes, but they are yet to be confirmed in nonlinear simulations. Valuable experimental insights in this direction are expected from high $\beta$ plasmas with $T_{\mathrm{e}} \sim T_{\mathrm{i}}$ at JT60-SA. Fast ions are found to provide significant stabilization in the strongly electromagnetic scenario both in terms of their equilibrium pressure and their dynamical role in the microturbulence. A caveat for scaling towards ITER is that the latter effect is presently studied best for NBI beam ions of the temperature $T_{f} \lesssim 10 T_{\mathrm{i}}$, while fusion $\alpha$ particles are much more energetic. Since dynamical effects are likely linked to certain phase-space resonances, future simulation studies will have to address the dependences on temperature, mass, and charge of the suprathermal species, as well their non-Maxwellian velocity distribution. Actual fusion $\alpha$ particles will be generated and studied during the scheduled JET D-T campaign, but already today, fast ion parameters can be accessed by using ICRH heating, possibly in addition to beam injection. At the same time this reduces the $E \times B$ flow shear. Such experiments would be extremely helpful to finally determine, whether the electromagnetic and fast-ion stabilization scenarios described in this paper can be expected in the plasmas of future machines.

\section{Acknowledgments}

The authors would like to thank C Angioni, A Di Siena, E Poli, M J Pueschel and D Told for valuable discussions.

This work has been carried out within the framework of the EUROfusion Consortium and has received funding from the Euratom research and training programme 2014-2018 under grant agreement No 633053. The views and opinions expressed herein do not necessarily reflect those of the European Commission.

The research leading to these results has received funding from the European Research Council under the European Unions Seventh Framework Programme (FP7/2007-2013)/ ERC Grant Agreement No. 277870.

The numerical results presented in this work were carried out using the HELIOS supercomputer system at the Computational Simulation Centre of International Fusion Energy Research Centre (IFERC-CSC), Aomori, Japan, under the Broader Approach collaboration between Euratom and Japan, implemented by Fusion for Energy and JAEA and using the resources of the RZG computing center, Garching, Germany.

\section{References}

[1] Sips A C C et al 2002 Plasma Phys. Control. Fusion 44 B69

[2] Luce T C et al 2003 Nucl. Fusion 43321

[3] Hobirk J et al 2012 Plasma Phys. Control. Fusion 54095001
[4] Challis C et al 2015 Nucl. Fusion 55053031

[5] ITER Physics Expert Group on Confinement and Transport et al 1999 Nucl. Fusion 392175

[6] Citrin J et al 2015 Plasma Phys. Control. Fusion 57014032

[7] Garcia J, Challis C, Citrin J, Doerk H, Giruzzi G, Görler T, Jenko F, Maget P and JET Contributors 2015 Nucl. Fusion 55053007

[8] Pueschel M J, Kammerer M and Jenko F 2008 Phys. Plasmas 15102310

[9] Pueschel M J and Jenko F 2010 Phys. Plasmas 17062307

[10] Citrin J, Jenko F, Mantica P, Told D, Bourdelle C, Garcia J, Haverkort J W, Hogeweij G M D, Johnson T and Pueschel M J 2013 Phys. Rev. Lett. 111155001

[11] Bravenec R et al 2015 APS Meeting Abstracts p. BAPS.2015. DPP.PP12.78

[12] Mailloux J 2015 APS Meeting Abstracts p. BAPS.2015.DPP. KI2.1

[13] Hill D and The DIII-D Team 2013 Nucl. Fusion 53104001

[14] Stroth U et al 2013 Nucl. Fusion 53104003

[15] Romanelli F and JET EFDA Contributors 2013 Nucl. Fusion 53104002

[16] Bourdelle C et al 2015 Nucl. Fusion 55063017

[17] Ishida S, Barabaschi P, Kamada Y and The JT-60SA Team 2011 Nucl. Fusion 51094018

[18] Kamada Y, Barabaschi P, Ishida S, The JT-60SA Team and JT-60SA Research Plan Contributors 2013 Nucl. Fusion 53104010

[19] Jenko F, Dorland W, Kotschenreuther M and Rogers B N 2000 Phys. Plasmas 71904

[20] Artaud J et al 2010 Nucl. Fusion 50043001

[21] Connor J W, Hastie R J and Taylor J B 1978 Phys. Rev. Lett. 40396

[22] Wesson J and Sykes A 1985 Nucl. Fusion 2585

[23] Xanthopoulos P, Mikkelsen D, Jenko F, Dorland W and Kalentev O 2008 Phys. Plasmas 15122108

[24] Tardini G et al 2007 Nucl. Fusion 47280

[25] Bourdelle C, Hoang G T, Litaudon X, Roach C M, Tala T, ITPA Topical Group on Transport and ITB Physics and International ITB Database Working Group $2005 \mathrm{Nucl}$. Fusion 45110

[26] Romanelli M, Zocco A, Crisanti F and JET EFDA Contributors 2010 Plasma Phys. Control. Fusion 52045007

[27] Holland C, Petty C C, Schmitz L, Burrell K H, McKee G R, Rhodes T L and Candy J 2012 Nucl. Fusion 52114007

[28] Joiner N, Hirose A and Dorland W 2010 Phys. Plasmas 17072104

[29] Kinsey J E, Waltz R E and Candy J 2005 Phys. Plasmas 12062302

[30] Bañón Navarro A, Teaca B, Jenko F, Hammett G W and Happel T 2014 Phys. Plasmas 21032304

[31] Jenko F, Dorland W and Hammett G W 2001 Phys. Plasmas 84096

[32] Sommer F et al 2015 Nucl. Fusion 55033006

[33] Scott B D 2005 Phys. Plasmas 12062314

[34] Pueschel M J, Terry P W, Jenko F, Hatch D R, Nevins W M, Görler T and Told D 2013 Phys. Rev. Lett. 110155005

[35] Terry P et al 2015 Nucl. Fusion 55104011

[36] Maeyama S, Ishizawa A, Watanabe T-H, Nakata M, Miyato N, Yagi M and Idomura Y 2014 Phys. Plasmas 21052301

[37] Maeyama S, Idomura Y, Watanabe T-H, Nakata M, Yagi M, Miyato N, Ishizawa A and Nunami M 2015 Phys. Rev. Lett. 114255002

[38] Howard N T, Holland C, White A E, Greenwald M and Candy J 2016 Nucl. Fusion 56014004

[39] Görler T and Jenko F 2008 Phys. Rev. Lett. 100185002

[40] Romanelli F et al 2015 Nucl. Fusion 55104001 